\title{
Theoretical Study of Transition Pressure and Volume Collapse on Samarium Chalcogenides $(\mathrm{SmX}, \mathrm{X}=\mathrm{S}, \mathrm{Se}$ and $\mathrm{Te})$
}

\author{
Dipak Raj Adhikari, Shanker Prasad Chimouriya \\ Department of Natural Sciences (Physics),Kathmandu University,Nepal
}

\begin{abstract}
In the present paper, we have been studied the theoretical transition pressure of samarium chalcogenides by using potential model. We have reviewed bulk modulus by simple calculation. The transition pressure, volume collapse and bulk modulus predicted from this approach are found to be close to experimental data.
\end{abstract}

Key words: Samarium Chalcogenides, phase transition, transition pressure.

\section{Introduction}

Single crystal or polycrystal of samarium chalcogenides can be obtained experimentally by reacting the metal with sulfur, selenium or tellurium vapors at high temperature [1]. Thin films can be obtained by magnetron sputtering [2] or electron beam physical vapor deposition i.e. bombardment of samarium metal target with electrons in an appropriate gas atmosphere (e.g. hydrogen disulfide for $\mathrm{SmS}$ ) [3]. The $\mathrm{SmS}$ returns to semiconducting state at lower pressure of about $0.5 \mathrm{kbar}$ [1]. In SmS not only color but also other properties are changed under the effect of pressure. The band gap of samarium chalcogenides are $0.15 \mathrm{eV}, 0.45 \mathrm{eV}$ and $0.65 \mathrm{eV}$ respectively at zero pressure [1-5]. In transition pressure (6.5 kbar for SmS) the gap is still finite and low resistivity. Samarium sulfide undergoes a discontinuous semiconductor to metal transition at appropriate pressure of $6.5 \mathrm{kbar}$.

\section{Theoretical Methodology}

In order to understand of some properties of SmX semiconducting compounds, we made following assumption: the change in force constant is small, the short-range interactions are effective up to the second neighbor ions, and the atoms are held together with harmonic elastic without any internal strain within the crystal. In this system, all ions are not at centers of inversion symmetry and undergo internal displacements when the lattice is strained. The strain does not change the macroscopic dimension of the material but involves the transition of the anion lattice relative cation lattice.The inter-ionic potential for the compound in the framework of two body interaction potential is expressed for the $\mathrm{SmX}$ in either $\mathrm{NaCl}$ or $\mathrm{CsCl}$ structure is given as

$U(r)=U_{C}(r)+U_{S R}(r)+U_{V}(r)$

The first term is being the Coulomb's energy, and follows as

$U_{C}(r)=-\sum_{i, j} \frac{Z_{i} Z_{j} e^{2}}{4 \pi \grave{\mathrm{o}}_{0} r_{i j}}=-\frac{\alpha_{m} Z^{2} e^{2}}{4 \pi \grave{\mathrm{o}}_{0} r}$

with $\alpha_{m}$ as the Madelung constant [6-8], $Z_{i}\left(Z_{j}\right)$ is the valency of cation(anion), $r_{i j}$ is the separation distance between $i$ and $j$ ions, $e$ is the electronic charge and $\grave{\mathrm{o}}_{0}$ is the permittivity in free space.

The second term of eqn.(1) represents the short-range (SR) overlap repulsive energy,

$$
\begin{aligned}
& U_{S R}(r)=\sum_{i, j} b \beta_{i j} \exp \left(\frac{r_{i}+r_{j}-r_{i j}}{\rho}\right) \\
& U_{S R}(r)=n_{1} b \beta_{i j} \exp \left(\frac{r_{i}+r_{j}-r}{\rho}\right)+\frac{n_{2}}{2} b \beta_{i i} \exp \left(\frac{2 r_{i}-k r}{\rho}\right)+\frac{n_{2}}{2} b \beta_{j j} \exp \left(\frac{2 r_{j}-k r}{\rho}\right)
\end{aligned}
$$

Following Hafemeister and Flygare potential, $k$ being the structure factor and $\beta_{i j}$ are the Pauling coefficient defined as:

$$
\beta_{i j}=1+\frac{Z_{i}}{n_{i}}+\frac{Z_{j}}{n_{j}}
$$


with $Z_{i}\left(Z_{j}\right)$ and $n_{i}\left(n_{j}\right)$ as the valency and number of outermost electrons in the cations(anion) respectively.

The symbol $b$ and $\rho$ are being the short-range ( $b$, hardness and $\rho$, range) repulsive potential parameters. The last term in eqn.(1) is the Van der Waal's (vdW) potential energy, expressed as:

$U_{V}(r)=-\sum_{i, j} \frac{c_{i j}}{r_{i j}^{6}}-\sum_{i, j} \frac{d_{i j}}{r_{i j}^{8}}=-\frac{C}{r^{6}}-\frac{D}{r^{8}}$

due to dipole-dipole (d-d) and dipole-quadrupole (d-q) interaction. $c_{i j}\left(d_{i j}\right)$ represents the Van der Waal coefficients associated to the $\mathrm{d}-\mathrm{d}(\mathrm{d}-\mathrm{q})$ interactions and $C(D)$ are the corresponding overall Van der Waal coefficients defined as [6-12]

$$
\begin{aligned}
& C=n_{1} c_{i j}+\frac{n_{2}}{2} \frac{c_{i i}+c_{j j}}{k^{6}} \\
& D=n_{1} d_{i j}+\frac{n_{2}}{2} \frac{d_{i i}+d_{j j}}{k^{8}}
\end{aligned}
$$

We follow the variational method (Slater and Kirkwood)[SKV Method]] [9-16] to derive $c_{i j}$ and $d_{i j}$ as [6]

$c_{i j}=\frac{3}{2} \frac{e \hbar}{\sqrt{m_{e}}}\left(\frac{1}{4 \pi \dot{\mathrm{o}}_{0}}\right)^{2} \alpha_{i} \alpha_{j}\left[\left(\frac{\alpha_{i}}{N_{i}}\right)^{1 / 2}+\left(\frac{\alpha_{j}}{N_{j}}\right)^{1 / 2}\right]^{-1}$

$d_{i j}=\frac{27}{8} \frac{\hbar^{2}}{m_{e}}\left(\frac{1}{4 \pi \grave{\mathrm{o}}_{0}}\right)^{2} \alpha_{i} \alpha_{j}\left[\left(\frac{\alpha_{i}}{N_{i}}\right)^{1 / 2}+\left(\frac{\alpha_{j}}{N_{j}}\right)^{1 / 2}\right]^{2} \times\left[\left(\frac{\alpha_{i}}{N_{i}}\right)+\frac{20}{3}\left(\frac{\alpha_{i} \alpha_{j}}{N_{i} N_{j}}\right)^{1 / 2}+\left(\frac{\alpha_{j}}{N_{j}}\right)\right]^{-1}$

where, $m_{e}$ is the electron's mass, $\alpha_{i}\left(\alpha_{j}\right)$ is the electronic polarizability and $N_{i}\left(N_{j}\right)$ denotes the effective number of electrons of the cation (anion). The values of overall Van der Waal coefficients are obtained using eqns. (6) and (7)

By substituting the expressions of $U_{C}(r), U_{S R}(r)$ and $U_{V}(r)$ in eq.(1) we obtain.

$$
\begin{aligned}
U(r)= & -\frac{\alpha_{m} Z^{2} e^{2}}{4 \pi \mathrm{̀}_{0} r}+n_{1} b \beta_{i j} \exp \left(\frac{r_{i}+r_{j}-r}{\rho}\right)+\frac{n_{2}}{2} b \beta_{i i} \exp \left(\frac{2 r_{i}-k r}{\rho}\right)+ \\
& \frac{n_{2}}{2} b \beta_{j j} \exp \left(\frac{2 r_{j}-k r}{\rho}\right)-\frac{C}{r^{6}}-\frac{D}{r^{8}}
\end{aligned}
$$

The bulk modulus for a given material is defined as

$$
B_{T}=-V \frac{d P}{d V}
$$

So at equilibrium condition, i.e. at zero pressure and zero Kelvin of temperature, the eqn. (10) becomes

$$
\left.\frac{d^{2} U(r)}{d r^{2}}\right|_{r=r_{0}}=9 K r_{0} B_{0}
$$

The change in volume associated with the electronic transition from divalent to trivalent state [17] is given by

$$
\left(\frac{\Delta V}{V_{0}}\right)_{\text {elec }}=\left[1+\left(\frac{\Delta a}{a_{0}}\right)\right]^{3}-1
$$

where $\Delta a=2\left(r^{+++}-r^{++}\right) \exp \left[-\left(\Delta E_{g}-\alpha P\right) / k T\right], r^{+++}$and $r^{++}$are the ionic radii of the samarium ion, $a_{0}$ and $\Delta E_{g}$ are the lattice parameter and $\alpha$ is the rate at which $\Delta E_{g}$ close with pressure. " $\alpha$ " the rate at which the energy gap $\Delta E_{g}$ between the $4 f$ level and conduction band decreases with pressure is determined 
[18] such as Anderson and Nafe [19] obtained and empirical relationship between bulk modulus $B_{0}$ at atmospheric pressure and specific volume (per ion pair) $V_{0}$ of the form given by

$$
B_{0} \sim\left(V_{0}\right)^{-x}
$$

They found for particular class of compounds where the value of $x$ depends on the class of compounds. For chalcogenides they found $x$ to be 1 . We have investigated as empirical relationship between bulk modulus $\left(B_{0}\right)$ at atmospheric pressure and specific volume $V_{0}$ given by

$B_{0}=A\left(V_{0}\right)^{-1}$

Where $A$ is the constant having the value $2 \times 10^{6}$. The values of lattice parameters $a$ and specific volume $V_{0}$ for samarium chalcogenides are given in table (6). Using the value of specific volume $V_{0}$, we have calculated bulk modulus $B_{0}$ for samarium chalcogenides. The calculated values of $B_{0}$ are compared with the experimental values and are found in a reasonable agreement between them, considering the error bars in the data available [11]. Over all calculation have been done by using MATLAB.

\section{Results and Discussion}

The objective of the study was to understand some of the thermodynamical variables associated with phase transition at zero temperature. The model has two free parameters: the range $(\rho)$ and hardness $(b)$, which was estimated by the proper utilization of the equilibrium distance. Deduced values of free parameters allowed us to predict the phase transition pressure and associated volume collapse. For the computational process first of all we have collected the structure dependent parameters; Madelung constant $\left(\alpha_{m}\right)$, number of nearest neighbor ions $\left(n_{1}\right)$, number of next nearest neighbor ions $\left(n_{2}\right)$, crystal constants $k$ and $K$ for $\mathrm{B} 1(\mathrm{NaCl})$ and $\mathrm{B} 2(\mathrm{CsCl})$ phases and listed these values in table1. The input data: the ionic radii of cation $\left(r_{i}\right)$ and anion $\left(r_{j}\right)$, equilibrium interionic separation $\left(r_{0}\right)$ at zero pressure, bulk modulus $\left(B_{0}\right)$ at zero pressure, electronic polarizabilities $\alpha_{i}\left(\alpha_{j}\right)$ of cation(anion) of SmS, SmSe and SmTe are presented in table 2. While performing the numerical computation, we presumed that the crystals considered are purely ionic, i.e. the interionic potential depends on the electronic polarizabilities of the individual ions.

Using the polarizabilities data of table 2, we first deduce the Van der Waal coefficients from the SlaterKirkood variation method using eqns. (8) and (9) as well as the overall Van der Waal coefficients using eqns. (6) and (7). The overall vdW coefficients of SmS, SmSe and SmTe for their B1 and B2 phases are presented in table 3. For such purposes, we have two free parameters, namely, range and hardness parameters ( $\rho$ and $b$ ). We have deduced their values from the knowledge of equilibrium distance and the bulk modulus following the equilibrium condition $\left.\frac{d U}{d r}\right|_{r=r_{0}}=0$ and eqn. (12). The values of $\rho$ and $b$ for SmS, SmSe and SmTe in their B1 and B2 phases are presented in table 4.In an attempt to reveal the structural phase transition of SmS, SmSe and SmTe, we minimize the Gibb's free energies $G_{B !}(r)$ and $G_{B 2}\left(r^{\prime}\right)$ for equilibrium interionic separation $(r)$ and $\left(r^{\prime}\right)$ for a given pressure. The Gibbs free energy difference $\Delta G=G_{B 1}(r)-G_{B 2}\left(r^{\prime}\right)$ have been plotted as function of pressure $(P)$ in Figure 1 for SmS, Figure 2 for SmSe and Figure 3 for SmTe by using the interionic potential discussed above. The pressure corresponding to $\Delta G \rightarrow 0$ is the phase transition pressure $\left(P_{t}\right)$ (indicated by arrow in figures). At zero pressure the B1 crystal phase is thermodynamically and mechanically stable, while B2 is not. As pressure increases, beyond the phase transition pressure $\left(P_{t}\right)$, the B2 system becomes mechanically and thermodynamically stable. We have also calculated the fractional volume collapse in percentage during the process of phase transition. The phase transition pressure $\left(P_{t}\right)$ and percentile fractional volume collapse $\left(\Delta V / V_{0}\right) \%$ are presented in table 5 along with available experimental data and obtained by other theoretical works. 
This concludes that the transition is almost continuous for SmSe and SmTe. It is thus argued that the major volume discontinuity in pressure-volume phase diagram for $\mathrm{SmS}$ identifies the structural phase transition from $\mathrm{NaCl}$ to $\mathrm{CsCl}$. We have also computed the value of relative volume associated with various compressions and plotted them against various pressures as shown in Figure 4 for SmS, Figure 5 for SmSe and Figure 6 for SmTe. We have also calculated the fractional volume collapse in percentage during the process of phase transition. The phase transition pressure $\left(P_{t}\right)$ and percentile fractional volume collapse $\left(\Delta V / V_{0}\right) \%$ and bulk modulus are presented in table 5 and 6 along with available experimental data and obtained by other theoretical works.

\section{References}

[1] A. Jayaraman, V. Narayanamurti, E. Bucher, and R. G. Mines, Phys. Rev. Letters1 25, 1430 (1970).

[2] R. Kitagawa, H. Takeba, and K. Mornaga, Appl. Phys. Letters 82, 3641 (2003).

[3] E. Rogars, P. F. Smell, P. Dorenbos, and E. V. D. Kolk, J. Phys. Condensed Matter 22, 015005 (2010).

[4] K. H. J. Buschow, Concise Encyclopedia of Magnetic and Supercondiuncting Material (2005), p. 318.

[5] C. M. Verma and V. Heine, Phys. Rev. 11, 4763 (1975).

[6] D. Varsney, R. Kinge, P. Sharma, N. Kaurav, and R. K. Singh, Indian J. Phys. 43, 939 (2005).

[7] M. P. Tosi and F. G. Fumi, J. Phys. Chem. Solids 23, 359 (1962).

[8] M. L. Huggins and J. E. Mayer, J. Chem. Solids 3, 637 (1935).

[9] J. E. Mayer, J. Chem. Solids 1, 270 (1933).

[10] J. C. Slater and J. G. Kirkood, Phys. Rev. 37, 682 (1931).

[11] U. Benedict and W. B. Holzafel, Handbook on the Physics and Chemistry of Rare Earths (North Holland, Amsterdam, 1993), p. 113 .

[12] V. A. Sidorov, N. N. Stepanov, L. G. Khvostantsev, O. B. Tsiok, A. V. Golubkove, V. S. Oskotsi, and I. A. Smirnov, Semicond. Sci. Technolq 4, 286 (1989).

[13] T. L. Bihan, S. Darracq, S. Heathman, U. Benedict, K. Mattenberger, and O. Vogt, J. Alloys. Compd. 226, 143 (1995).

[14] O. B. Tsiok, V. A. Sidorov, V. V. Bredikhin, L. G. Khvostantsev, A. V. Golubkove, and I. A. Smirnov, Solid State Commun. 79, 227 (1991).

[15] W. M. Temmarman, A. Svane, L. Petit, M. Luders, P. Strange, and Z. Szotek, Phase Transition 80, (2007)

[16] D. Varsney, N. Kaurav, P. Sharma, S. Shan, and R. K. Singh, Phase Transition2 77, 1075 (2004).

[17] M. Francilon, D. Gerome, J. C. Achard, and G. Mailfait, J. Phys (Paris) 31, 709 (1970).

[18] E. Bucher, V. Narayanamurti, and A. Jayaraman, J. Appl. Phys. 42, 1741 (1971).

[19] O. L. Anderson and J. L. Nafe, J. Geophys. Res 70, 3951 (1965).

Table 1. The structure dependent input parameters: Madelung constant $\left(\alpha_{m}\right)$, number of nearest and next

\begin{tabular}{llllll}
\multicolumn{5}{c}{ nearest neighbor ions $\left(n_{1}\right)$ and $\left(n_{2}\right)$, crystal constants $(k)$ and $(K)}$. \\
\hline Phase & $\alpha_{m}$ & $n_{1}$ & $n_{2}$ & $k$ & $K$ \\
B1 $(\mathrm{NaCl})$ & 1.7475 & 6 & 12 & $\sqrt{2}$ & 2 \\
B2(CsCl $)$ & 1.7626 & 8 & 6 & $2 / \sqrt{3}$ & $8 /(3 \sqrt{3})$ \\
\hline
\end{tabular}

Table 2. The input parameters: ionic radius of cation $\left(r_{i}\right)$, ionic radius of anion $\left(r_{j}\right)$, equilibrium interionic separation $\left(r_{0}\right)$, electronic polarizability of cation $\left(\alpha_{i}\right)$, electronic polarizability of anion $\left(\alpha_{j}\right)$, bulk modulus at zero pressure $\left(B_{0}\right)$ for $\mathrm{SmS}, \mathrm{SmSe}$ and SmTe.

\begin{tabular}{llllllll}
\hline Compound & $r_{i}(\AA)$ & $r_{j}(\AA)$ & $a(\AA)$ & $r_{0}(\AA)$ & $\alpha_{i}\left(\AA^{3}\right)$ & $\alpha_{j}\left(\AA^{3}\right)$ & $B_{0}(\mathrm{kbar})$ \\
& $1.36^{\mathrm{b}}$ & $1.70^{\mathrm{b}}$ & $5.97^{\mathrm{a}}$ & 4.221 & $28.8^{\mathrm{b}}$ & $2.9^{\mathrm{b}}$ & $476^{\mathrm{a}}$ \\
$\mathrm{SmS}$ & $1.36^{\mathrm{b}}$ & $1.84^{\mathrm{b}}$ & $6.22^{\mathrm{a}}$ & 4.398 & $28.8^{\mathrm{b}}$ & $3.8^{\mathrm{b}}$ & $520^{\mathrm{a}}$ \\
$\mathrm{SmTe}$ & $1.36^{\mathrm{b}}$ & $2.07^{\mathrm{b}}$ & $6.60^{\mathrm{a}}$ & 4.662 & $28.8^{\mathrm{b}}$ & $5.5^{\mathrm{b}}$ & $400^{\mathrm{a}}$ \\
\hline
\end{tabular}

${ }^{a} \operatorname{Ref}[5],{ }^{b} \operatorname{Ref}[15,16]$

Table 3. Calculated over all Van der Waal coefficients $C$ and $D$ of $\mathrm{SmS}, \mathrm{SmSe}$ and SmTe for B1 and B2 phases

\begin{tabular}{llccc}
\hline & B1(NaCl) structure & \multicolumn{3}{c}{ B2(CsCl) structure } \\
Compound & $C\left(\mathrm{eV \AA}^{6}\right)$ & $D\left(\mathrm{eV} \mathrm{\AA}^{8}\right)$ & $C\left(\mathrm{eV}^{6}\right)$ & $D\left(\mathrm{eV}^{8}\right)$ \\
SmS & 2904 & 10697 & 5172 & 18319 \\
$\mathrm{SmSe}$ & 3344 & 12605 & 5840 & 20825 \\
$\mathrm{SmTe}$ & 4134 & 16145 & 7030 & 25542 \\
\hline
\end{tabular}


Table 4. Computed model parameters: range parameter $(\rho)$ and hardness parameter $(b)$ of SmS, SmSe and SmTe for B1 and B2 phases.

\begin{tabular}{lllll}
\hline \multirow{2}{*}{ Compound } & $\mathrm{B} 1(\mathrm{NaCl})$ structure & & $\mathrm{B} 2(\mathrm{CsCl})$ structure \\
& $\rho(\AA)$ & $b(\mathrm{eV})$ & $\rho(\AA)$ & $b(\mathrm{eV})$ \\
$\mathrm{SmS}$ & 0.2237 & 23.6972 & 0.2887 & 5.4404 \\
$\mathrm{SmSe}$ & 0.6317 & 7.6837 & 0.7451 & 3.8292 \\
$\mathrm{SmTe}$ & 0.6938 & 6.3525 & 0.8075 & 3.2811 \\
\hline
\end{tabular}

Table 5. The calculated values of phase transition pressure $\left(P_{t}\right)$ and percentile volume collapse $\left(\Delta V / V_{0}\right)$ for SmS, SmSe and SmTe.

\begin{tabular}{lllll}
\hline & $P_{t}(\mathrm{kbar})$ & & $\Delta V / V_{0}(\%)$ \\
Compounds & $\begin{array}{l}\text { Present } \\
\text { (Potential }\end{array}$ & Others' works & $\begin{array}{l}\text { Present } \\
\text { (Potential } \\
\text { model) }\end{array}$ & Others' work \\
& model) & & 11.71 & $13.5^{\mathrm{a}}, 13.8^{\mathrm{b}}, 11.1^{\mathrm{e}}$ \\
$\mathrm{SmS}$ & 6.98 & $6.5^{\mathrm{a}}, 12.4^{\mathrm{b}}, 10^{\mathrm{e}}$ & 0.27 & $8^{\mathrm{a}}, 11^{\mathrm{c}}, 7^{\mathrm{d}}, 9.8^{\mathrm{e}}$ \\
$\mathrm{SmSe}$ & 76.5391 & $40^{\mathrm{a}}, 34^{\mathrm{b}}, 30-90^{\mathrm{c}}, 26-40^{\mathrm{d}}, 33^{\mathrm{e}}$ & 0.28 & $9^{\mathrm{c}}, 7^{\mathrm{d}}, 8.4^{\mathrm{e}}$ \\
\hline SmTe & 60.0527 & $20-80^{\mathrm{a}}, 52^{\mathrm{b}}, 60-80^{\mathrm{c}}, 46-75^{\mathrm{d}}, 62^{\mathrm{e}}$ & $\mathrm{e}^{\mathrm{e}}$ & 0.28 \\
\hline
\end{tabular}

${ }^{\mathrm{a}}$ Ref. [11], ${ }^{\mathrm{b}}$ Ref. [12], ${ }^{\mathrm{c}}$ Ref. [13], ${ }^{\mathrm{d}}$ Ref. [14], ${ }^{\mathrm{e}}$ Ref. [15]

Table 6. Values of lattice parameter and bulk modulus for divalent samarium chalcogenides

\begin{tabular}{lllll}
\hline Compounds & Lattice parameter & Specific volume & Bulk modulus & Bulk modulus \\
& $(a) \AA$ & $(M / \rho) \mathrm{cm}^{3}$ & $\left(B_{0}\right) \mathrm{kbar}$ & $\left(B_{0}\right) \mathrm{kbar}$ \\
& & & Calc & Expt. \\
$\mathrm{SmS}$ & 5.97 & 32.0 & 628 & 476 \\
$\mathrm{SmSe}$ & 6.22 & 35.86 & 556 & 520 \\
$\mathrm{SmTe}$ & 6.60 & 43.2 & 462 & 400 \\
\hline
\end{tabular}

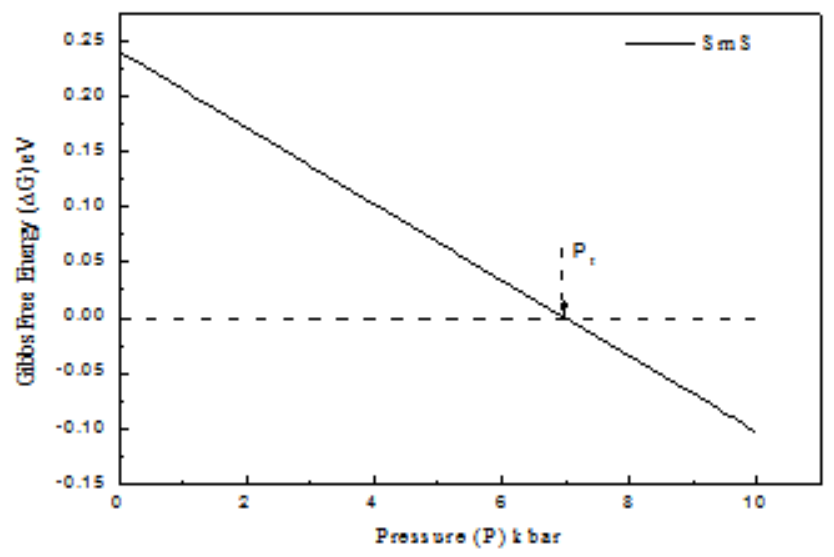

Figure 1. The variation of Gibb's free energy difference with pressure for $\mathrm{SmS}$

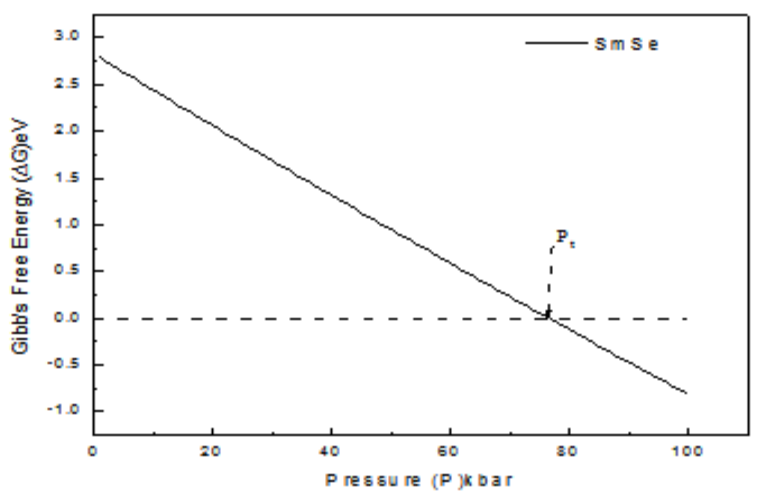

Figure 2. The variation of Gibb's free energy difference with pressure for SmSe 


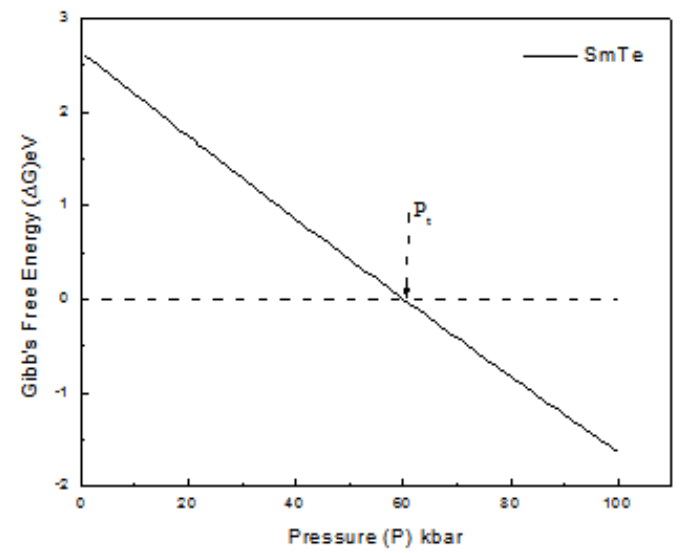

Figure 3. The variation of Gibb's free energy difference with pressure for SmTe

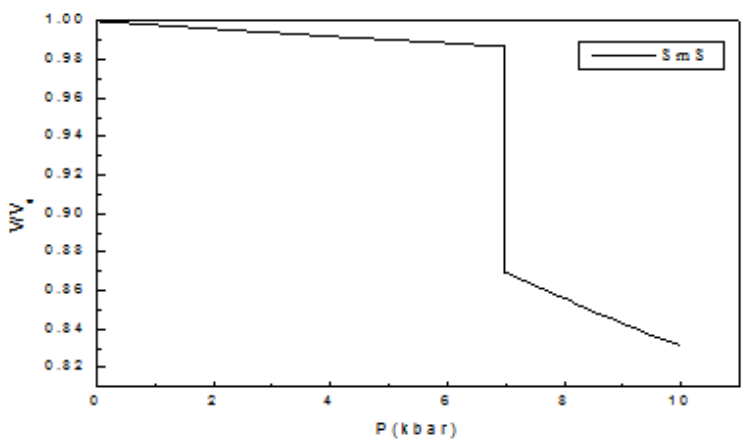

Figure 4. The variation of fractional volume with pressure for SmS

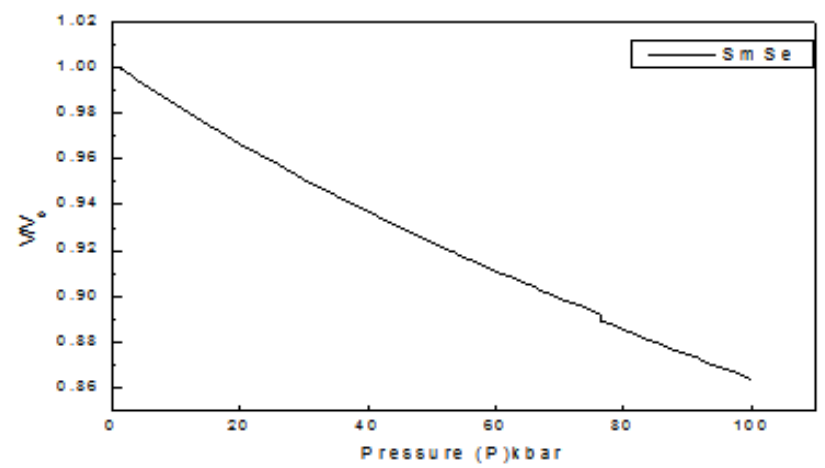

Figure 5. The variation of fractional volume with pressure for $\mathrm{SmSe}$

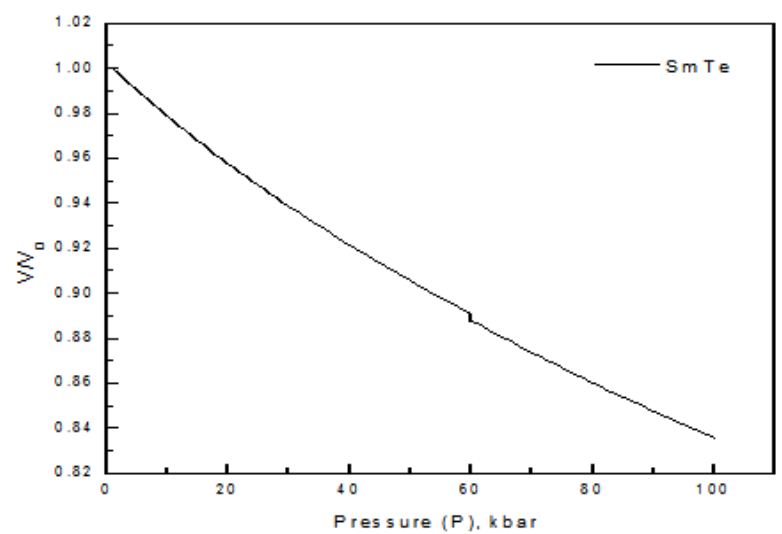

Figure 6. The variation of fractional volume with pressure for $\mathrm{SmTe}$ 